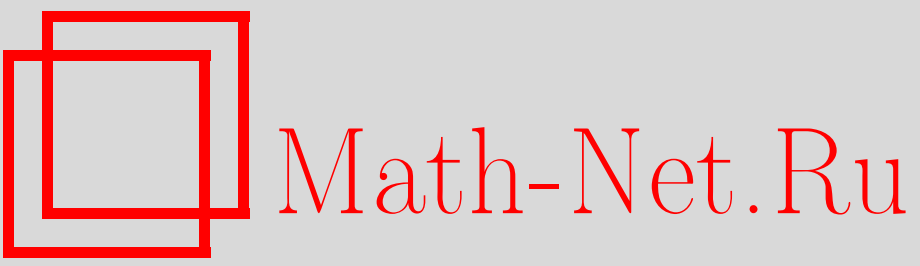

К. А. Агахи, Ю. Г. Басалов, В. Н. Кузнецов, Л. В. Фомин, Моделирование процесса ползучести с учётом стадии предразрушения и идентификация модели, Вестн. Сам. гос. техн. ун-та. Сер. Физ.-мат. науки, 2009, выпуск 2(), 243-247

DOI: https://doi.org/10.14498/vsgtu711

Использование Общероссийского математического портала Math-Net.Ru подразумевает, что вы прочитали и согласны с пользовательским соглашением

http://www. mathnet.ru/rus/agreement

Параметры загрузки:

IP : 54.81 .137 .203

26 апреля 2023 г., 14:56:44 
Механика деформируемого твёрдого тела

\author{
УДК 539.376
}

\title{
МОДЕЛИРОВАНИЕ ПРОЦЕССА ПОЛЗУЧЕСТИ С УЧЁТОМ СТАДИИ ПРЕДРАЗРУШЕНИЯ И ИДЕНТИФИКАЦИЯ МОДЕЛИ
}

\author{
К. А. Агахи, Ю. Г. Басалов, В. Н. Кузнецов, Л. В. Фомин
}

НИИ механики МГУ им. М. В. Ломоносова,

119992, Москва, Мичуринский пр-т, д. 1.

E-mails: kamilla@imec.msu.ru, basalov@yandex.ru,

vnkuznetsov@imec.msu.ru, lef1975@rambler.ru

При моделировании стадии предразрушения в прочессе ползучести обычно используется функиия повреждённости Работнова, для которой постулируется дифференииальное уравнение. В настоящей работе функиия повреждённости впервые определена непосредственно из серии опытов на ползучесть. Предложена новая модель процесса ползучести с учётом стадии возрастающей скорости, использующая интегральный оператор типа нормы Лебега и функиию нестабильности, определяемую экспериментально.

Ключевые слова: ползучесть, функиия повреждённости, моделирование, эксперимент, идентификаиия, обобщённая модель, интегральный оператор, норма Лебега.

Введение. В настоящей работе рассматривается вопрос моделирования ползучести при постоянном напряжении, причём особенное внимание уделено стадии предразрушения или прогрессирующей ползучести, когда в экспериментах на ползучесть наблюдается деформация с возрастающей скоростью.

Классические результаты в моделировании стадии прогрессирующей ползучести принадлежат Ю.Н. Работнову [1] и Л.М. Качанову [2], предположившим механизм возрастания скорости деформации, связанный с развитием и быстрым ростом повреждённости материала, которая, предположительно, имела смысл возникновения и быстрого «размножения» микротрещин. В работе [1] предложено учитывать данный механизм, используя известное уравнение ползучести $\dot{p}=A_{0} \sigma^{k}$ для установившейся стадии, модифицированное с помощью функции повреждённости $\omega$, которая должна определяться из специальных опытов (например, из структурных исследований) на стадии предразрушения с целью установления и описания процесса накопления и роста микротрещин, ослабляющих рабочее сечение. Однако проведение опытов по измерению повреждённости до сих пор остаётся нерешённой задачей.

1. Модель Ю. Н. Работнова. Основное уравнение теории ползучести имеет вид [1]

$$
\dot{p}=\frac{A_{0} \sigma^{k}}{1-\omega}
$$

причём $\omega=1$ в момент разрушения. Для определения функции повреждённости

Камилла Абдул Гусейн кызы Агахи (к.ф.-м.н., доц.), старший научный сотрудник, лаборатория прочности и ползучести при высоких температурах. Юрий Генрихович Басалов, ведущий инженер, лаборатория прочности и ползучести при высоких температурах. Владимир Николаевич Кузнецов (д.ф.-м.н., доц., ак-к. РАЕН), зав. лабораторией, лаборатория прочности и ползучести при высоких температурах. Леонид Викторович Фомин, ведущий инженер, лаборатория прочности и ползучести при высоких температурах. 
$\omega(t)$ постулировано дифференциальное уравнение первого порядка

$$
\dot{\omega}=\frac{B_{0} \sigma^{m}}{(1-\omega)^{s}},
$$

и, таким образом, законы ползучести представляются соотношениями (1) и (2), где $p=\varepsilon-\varepsilon_{0}-$ деформация ползучести; $\varepsilon-$ текущая деформация; $\varepsilon_{0}-$ начальная «мгновенная» деформация в момент нагружения; $\dot{p}=d p / d t-$ производная деформации ползучести $p$ по времени $t$; $\sigma=$ const - напряжение, приложенное к образцу; $\omega=\omega(t)$ - функция повреждённости; $A_{0}, B_{0}, m, k, s$ - константы модели.

Для идентификации модели (1) и (2) использовался следующий алгоритм: решалось дифференциальное уравнение первого порядка (2) и находилась функция $\omega=\omega\left(t, \sigma, A_{0}, B_{0}, m, s\right)$; затем данное решение подставлялось в $(1)$, получалось аналитическое выражение для $\dot{p}$, а затем и для $p$; далее, подбирая соответствующие константы модели из имеющихся опытов, можно максимально близко описать экспериментальные данные. Однако подобрать константы, обеспечивающие приемлемую точность, нелегко, по-видимому, по причине несогласованности уравнения (2) с уравнением (1).

2. Прямой экспериментальный метод идентификации модели. В настоящей работе предложен новый метод идентификации модели (1), (2) непосредственно из серии опытов на ползучесть без постулирования уравнения для функции повреждённости. Такой подход позволяет избежать введения в модель не опирающегося на эксперимент уравнения и связанных с этим трудностей.

Авторами предлагается следующий подход. Используя уравнение (1), находим функцию поврежденности непосредственно из опыта следующим образом. Получив из эксперимента с заданным уровнем напряжений $\sigma$ деформациию $p$ как функцию времени $p=p(t)$, определим производную деформации $\dot{p}(t)$. Зная $\dot{p}(t)$, найдём из $(1)$ функцию повреждённости $\omega_{i}=\omega\left(t, \sigma_{i}\right)$ в виде

$$
\omega\left(t, \sigma_{i}\right)=1-\frac{A_{0}\left(\sigma_{i}\right)^{k}}{\dot{p}(t)} .
$$

Таким образом, функция $\omega_{i}=\omega\left(t, \sigma_{i}\right)$ определяется из эксперимента с точностью, равной точности экспериментальных данных для каждой кривой семейства. На рис. 1 представлено семейство экспериментальных кривых [3] для меди после термообработки (отжиг в вакууме $1,3 \cdot 10^{-8}$ атм в течении двух часов при температуре $800{ }^{\circ} \mathrm{C}$ и последующее охлаждение с печью) при температуре $T=400^{\circ} \mathrm{C}$.

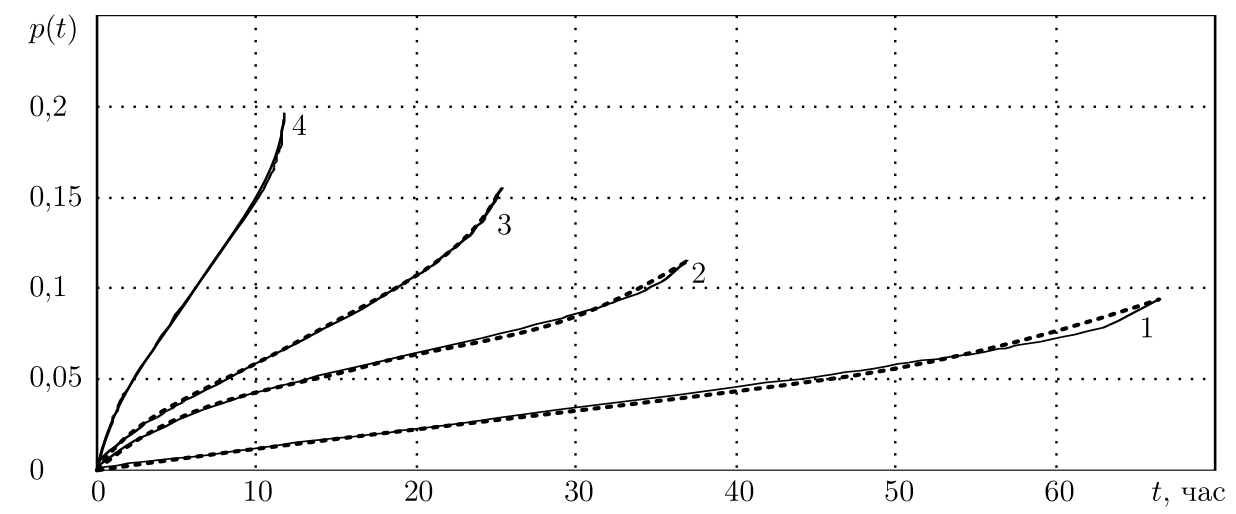

Рис. 1. Семейство кривых ползучести (пунктирная линия - эксперимент, сплошная - аппроксимация): $1-\sigma=40$ МПа, $2-\sigma=50$ МПа, $3-\sigma=60 \mathrm{MПа,} 4-\sigma=70$ МПа 
В нашем случае задача моделирования состоит в том, чтобы полностью вычислить кривую ползучести при произвольном уровне напряжения $\sigma$ в известном диапазоне на основании имеющихся экспериментальных данных для конкретного материала. ритм.

Для решения сформулированной задачи предлагается нижеследующий алго-

Все имеющиеся кривые ползучести аппроксимируются зависимостью одного вида для всех кривых, при условии, что значения коэффициентов аппроксимации зависят от уровня напряжения $\sigma$. Для кривых, представленных на рис. 1 , хорошее приближение даёт следующая аппроксимация:

$$
p(t)=A t+B(1-\exp (-\lambda t))+\frac{C}{\left(t_{*}-t\right)^{n}},
$$

где $t_{*}>t_{\max }, t_{\max }$ - время, соответствующее разрушению образца (значение $t_{*}$ выбирается из условия наилучшей аппроксимации для каждой кривой). Коэффициенты аппроксимации (4) $A, B, C, n, \lambda, t_{*}$ вычисляются для каждой кривой ползучести семейства, представленных на рис. 1, методом наименьших квадратов.

Структура выбранной аппроксимации (4) естественным образом описывает все три стадии ползучести, причём коэффициенты $A, B, C, n, \lambda, t_{*}$ зависят только от напряжения $\sigma$ (при заданной температуре). Тогда, имея некоторое количество опытов для разных уровней напряжений и определив для каждого опыта коэффициенты $A$, $B, C, n, \lambda, t_{*}$, можно построить таблицу их значений.

Зависимость коэффициентов аппроксимации от напряжения для кривых,

\begin{tabular}{|c|c|c|c|c|c|}
\hline \multicolumn{2}{|c|}{$\sigma$, МПа } & 40 & 50 & 60 & 70 \\
\hline & $A$ & 0,00109 & 0,00174 & 0,0041 & 0,01206 \\
\hline I & $B$ & 0 & 0,02618 & 0,01221 & 0,02415 \\
\hline 寻 & $C$ & 0,07848396 & 0,07492052 & 0,10226493 & 0,00829872 \\
\hline ? & $n$ & 1,0 & 1,0 & 1,0 & 1,0 \\
\hline क्ष & $\lambda$ & 0,0 & 0,2 & 0,4 & 1,0 \\
\hline ث & $t_{*}$ & 70 & 40 & 28 & 12 \\
\hline
\end{tabular}

Пользуясь таблицей, можно построить графики для величин $A, B, C, n, \lambda, t_{*}$, считая, что $A=A(\sigma), B=B(\sigma), C=C(\sigma), n=n(\sigma), \lambda=\lambda(\sigma), t_{*}=t_{*}(\sigma)-$

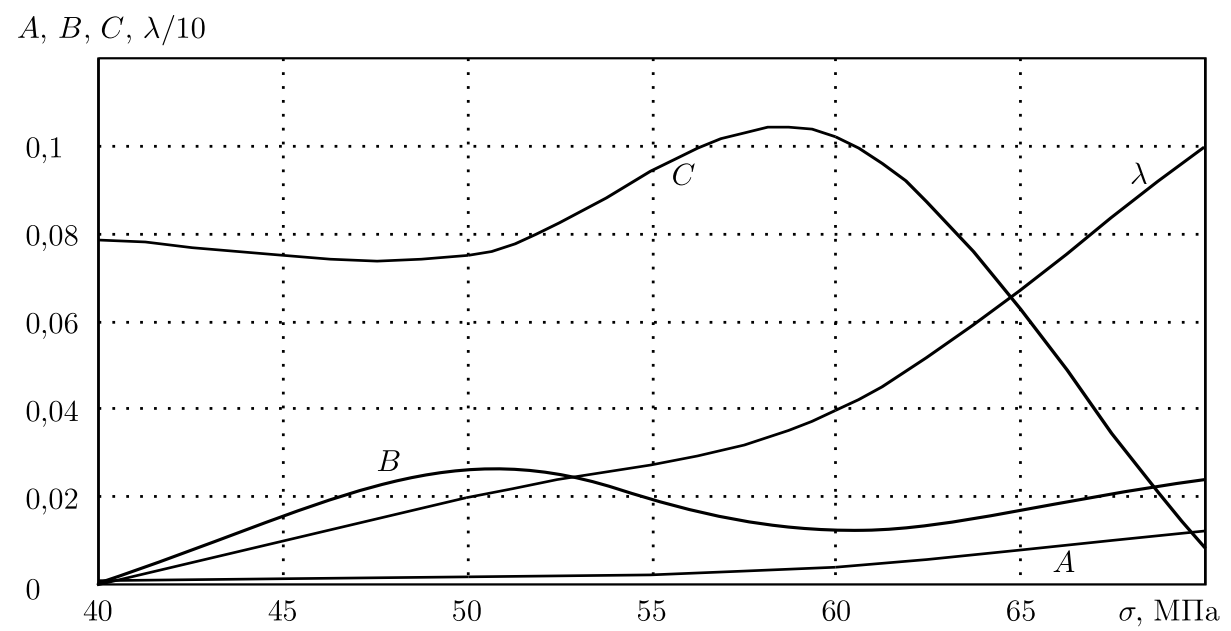

Рис. 2. Аппроксимации коэффициентов $A, B, C \lambda$ от $\sigma$ 
гладкие функции. По аппроксимациям этих величин можно найти соответствующие коэффициенты в формуле (4) для любого прогнозируемого опыта в заданном диапазоне напряжений $\sigma$ (зависимость коэффициентов от $\sigma$ представлены на рис. 2 ). Совокупность формул (1), (4) и зависимостей для $A, B, C, n, \lambda, t_{*}$ от $\sigma$ полностью определяет математическую модель процесса ползучести при заданной температуре $T$. Для других значений температур задача решается аналогично.

Имея данные аппроксимации, подставив $p$ из (4) в уравнение (3), получим аналитическое выражение для $\omega$, которая будет определена для любого опыта в известных пределах точности.

Отметим, что в основе построенного решения лежит аппроксимация кривых ползучести, причём возможны разные конкретные реализации аппроксимации. Возможно также прямое использование оцифровки, что позволяет всегда иметь точность, равную точности эксперимента, однако аналитическое представление позволяет получить интересный, наглядный и удобный результат.

3. Реологическая модель ползучести с учётом стадии предразрушения. Подход к описанию стадии предразрушения с помощью функции повреждённости можно осуществить, используя реологическую модель нестабильного материала, имеющую более общий характер в том смысле, что она позволяет описать процессы ползучести при переменном напряжении, процесс релаксации, «быстрые» процессы нагружения с постоянной скоростью и т. п. Вариант этой модели с одной функцией нестабильности для описания процессов ползучести имеет вид

$$
\varepsilon(t)=B|\bar{\sigma}(t)|^{\mu-1}\left(\int_{0}^{t}|\bar{\sigma}(\tau)|^{r} \hat{\psi}(\tau, \sigma) d \tau\right)^{\beta} \bar{\sigma}(t),
$$

где $B, \mu, r, \beta$ - константы материала, для определения которых используются стандартные опыты на растяжение с постоянной скоростью и опыты на ползучесть.

Функция $\bar{\sigma}(t)$ определяется следующим образом. Если материал в «быстрых» опытах подчиняется закону Гука, то $\bar{\sigma}=\sigma / E$, где $E-$ характерное значение модуля упругости. Если в указанных опытах имеет место нелинейность $\varepsilon=\Phi(\sigma)$, то имеем функцию $\bar{\sigma}=\Phi(\sigma)$, описывающую кривую растяжения при характерном значении скорости нагружения.

Функция нестабильности $\hat{\psi}(t, \sigma)$ играет роль ядра интегрального оператора и может быть определена из опытов на ползучесть при $\sigma=$ const $(\bar{\sigma}=$ const). В этом случае из (5) имеем

$$
\hat{\psi}(t, \sigma)=\frac{1}{B^{1 / \beta}|\bar{\sigma}(t)|^{(\mu+r \beta) / \beta}} \frac{d}{d t}\left(\varepsilon(t)^{1 / \beta}\right) .
$$

Применяя описанную выше процедуру аппроксимации к функции $\varepsilon(t)$ с коэффициентами, зависящими от $\sigma$, строим искомую функцию $\hat{\psi}(t, \sigma)$, что замыкает задачу моделирования процесса ползучести с учётом стадии предразрушения.

Покажем, что модель (1), (3) получается как частный случай модели (5), (6). Действительно, при $\beta=1$ и $\bar{\sigma}=\sigma / E$ с учётом $\dot{\varepsilon}(t)=\dot{p}(t)$ из (5) получаем

$$
\dot{p}=A \sigma^{\mu+r} \hat{\psi}(t, \sigma) .
$$

Поскольку из (1) имеем $\dot{p}=A_{0} \sigma^{k} /(1-\omega)$, то, сравнивая последние два выражения и полагая $\mu+r=k$, получаем

$$
\hat{\psi}(t, \sigma)=[1-\omega(t, \sigma)]^{-1} .
$$

При этом условии модели (7) и (3) совпадают.

Заключение. Отметим следующие основные результаты работы. 
1. Предложен новый метод идентификации известной модели процесса ползучести Ю. Н. Работнова, основанный на прямом использовании экспериментальных данных для определения функции повреждённости.

2. Предложена реологическая модель процесса ползучести, основанная на использовании интегрального оператора типа нормы Лебега, учитывающая возрастание скорости ползучести на стадии предразрушения.

Работа выполнена при поддержке РФФИ (№№ 07-01-00558, 08-08-00704). Авторы выражают благодарность А. М. Локощенко за полезные обсуждения и внимание к работе.

\section{БИБЛИОГРАФИЧЕСКИЙ СПИСОК}

1. Работнов Ю. Н. Ползучесть элементов конструкций. - М.: Наука, 1966. - 752 с.

2. Качанов Л. М. Теория ползучести. - М.: Физматгиз, 1960. - 456 с.

3. Локощенко A. М. Моделирование процесса ползучести и длительной прочности металлов. - М.: МГИУ, 2007. - 264 с.

Поступила в редакцию 20/VII/2009;

в окончательном варианте - 09/IX/2009.

\section{MSC: $74 \mathrm{C05}$}

\section{CREEP PROCESS SIMULATION CONSIDERING PRE-DESTRUCTION STAGE AND MODEL IDENTIFICATION}

\section{K. A. Agakhi, Yu. G. Basalov, V. N. Kuznetsov, L. V. Fomin}

Research Institute of Mechanics, M. V. Lomonosov Moscow State University, 1, Michurinsky pr., Moscow, 119192.

E-mails: kamilla@imec.msu.ru, basalov@yandex.ru,

vnkuznetsov@imec.msu.ru, lef1975@rambler.ru

It was suggested to define Rabotnov's function (damage function) from the differential equation of the first order for simulation of a pre-destruction stage in creep process. In this paper, the damage function is for the first time determined directly from a series of creep tests, independently of the formulated differential equation which, generally speaking, does not concord with the experimental data. A non-linear model of the creep process is suggested taking into consideration the rate increase stage, using an integral operator as Lebega's norm, and containing a damage function determined experimentally.

Key words: creep, damage function, modeling, experiment, identification, generalized model, integral operator, norm of Lebeg.

Original article submitted 20/VII/2009; revision submitted 09/IX/2009.

Kamilla A. Agahi (Ph. D. (Phys. \& Math.)), Senior Scientific Researcher, Laboratory of Durability and Creep at Heats. Yuriy G. Basalov, Leading Engineer, Laboratory of Durability and Creep at Heats. Vladimir N. Kuznetsov (Dr. Sci. (Phys. \& Math.), Academician of the Russian Academy of Natural Sciences), Head of Laboratory, Laboratory of Durability and Creep at Heats. Leonid V. Fomin, Leading Engineer, Laboratory of Durability and Creep at Heats. 